\title{
Classificação de Objetos de Aprendizagem Segundo o Grau de Multimodalidade
}

\author{
Alberto Bastos do Canto Filho - alberto.canto@ufrgs.br - PPGIE - UFRGS \\ Thaísa Jacintho Müller - thaisa.muller@pucrs.br - PPGIE - UFRGS - PUCRS \\ Érico M. H. Amaral - erico.amaral@unipampa.edu.br - PPGIE - UFRGS -UNIPAMPA \\ José Valdeni de Lima - valdeni@inf.ufrgs.br - PPGIE - UFRGS \\ Liane Margarida Rockenbach Tarouco - liane@penta.ufrgs.br - PPGIE - UFRGS
}

Resumo: Este artigo apresenta o extrato de uma pesquisa que avalia os níveis de multimodalidade inerentes a objetos de aprendizagem e propõe um padrão para classificação de softwares educacionais de acordo com os tipos e quantidades de mídias presentes. Para o desenvolvimento do estudo, alguns conceitos como estilo individual, carga cognitiva e multimodalidade foram amplamente explorados. Também foram observados os recursos comumente disponíveis em aplicações educacionais, como: Audio Verbal e Não Verbal, Visual Verbal e Não Verbal e Interação, os quais originaram, inicialmente, uma classificação de 18 pontos, que foram reduzidos a 7 classes. Para validação desta pesquisa, foi realizado um experimento com uma turma de graduação, o qual serviu para corroborar com a efetividade da classificação criada.

Palavras Chave - multimodalidade, classificação, estilo cognitivo

Abstract: This paper represents the extract of a research that evaluates the levels of multimodality related to learning objects and proposes a standard for rating educational softwares according to the kind and the amount of media contained in them. For developing this research some concepts like personal style, cognitive charge and multimodality have been broadly explored, resources ordinarily avaiable in educational applications were observed too, like: Audio Verbal and Nonverbal, Visual Verbal and Nonverbal and interaction, which originated, initially, a classification of 18 points, that were reduced to 7 grades. In order to validate this research, an experiment was made, applied to a graduation class, which presented effectual information that cooperated with the effectiveness of the classification created.

Keywords - multimodality, classification, cognitive style

\section{Introdução}

Com a disseminação dos Objetos de Aprendizagem (OAs) no meio educacional, faz-se necessário que se estabeleçam critérios de qualidade que simplifiquem não apenas a especificação e desenvolvimento destes objetos, mas também a seleção de objetos de aprendizagem adequados aos objetivos educacionais de professores, alunos e demais interessados.

As pesquisas desenvolvidas por Mussoi, Flores e Behar (2010), indicam parâmetros a serem observados na avaliação de objetos de aprendizagem, tais como a concepção epistemológica, a qualidade do conteúdo, a adequação do conteúdo ao público alvo/faixa etária, a definição de objetivos a serem alcançados, a forma de feedback ao usuário, a motivação, a forma de apresentação (layout, navegação, usabilidade) e a reusabilidade. 
Um objeto de aprendizagem deve ser eficaz ${ }^{1}$ e, por isso, tanto o projeto como a seleção de materiais existentes precisa passar por algum tipo de avaliação desta eficácia. A medida direta da eficácia necessita não apenas de uma clara definição de objetivos educacionais (Bloom, 1956), mas também de um processo que seja capaz de mensurar a proporção em que estes objetivos são alcançados quando se utiliza um determinado OA. Ocorre que, embora a qualidade do OA seja um fator relevante para a eficácia educacional, há outros fatores que podem preponderar. A utilização de material potencialmente significativo é apenas uma das condições para o aprendizado significativo (Ausubel, 1978). Em outras palavras, a medida direta da eficácia é um processo complexo, que envolve um grupo de alunos representativo do público alvo e a avaliação do aprendizado a partir do uso OA. Além do elevado custo de avaliação, a mensuração da eficácia somente poderá ser feita após o desenvolvimento do OA, o que inviabiliza a utilização direta da grandeza eficácia durante a etapa de projeto, levando à necessidade de que se estabeleçam critérios de mensuração indireta, como, por exemplo, a verificação se determinados princípios de projeto são respeitados. Esta verificação pode ser realizada de uma forma simples para determinados princípios, tais como constatar a continuidade temporal e espacial a coerência ou o nível de interatividade. Em outros casos, como a verificação da multimodalidade, a avaliação pode se tornar mais complexa, devido às diversas formas de multimodalidade possíveis, algumas das quais com potencial ganho de eficácia e outras com prejuízo.

A pesquisa aqui relatada propõe um sistema de classificação da multimodalidade e foi organizada da seguinte forma: em um primeiro momento foi feita uma revisão bibliográfica sobre questões de multimodalidade relacionadas aos processos de aprendizagem, apresentada na seção 2. As seções 3, 3.1 e 3.2 trazem uma primeira discussão sobre a proposta do método criado para classificação, o qual foi aprimorado conforme descrito na seção 3.3. Em seguida, o modelo criado passou por uma primeira fase de testes e validação, apresentada na seção 3.4. Por fim, na seção 4, são colocadas algumas considerações finais e perspectivas futuras.

\section{Referencial Teórico}

Dando início à apresentação da teoria estudada, destaca-se que a multimodalidade será definida aqui como a característica dos ambientes de aprendizagem que utilizam simultaneamente dois modos de apresentação: verbal e não verbal (Paivio, 1986). A eficácia dos sistemas multimodais tem sido fundamentada em estudos sobre os estilos individuais dos estudantes e na teoria da carga cognitiva (Sweller, 1988), como será apresentado nas seções a seguir.

\subsection{Estilos Individuais}

Felder e Brent (2005) definem estilo de aprendizagem como a forma característica com que os estudantes tomam e processam a informação. Grimley e Riding (2009) utilizam a expressão estilo cognitivo para designar a abordagem que o indivíduo normalmente utiliza quando processa uma informação. Uma abordagem comum entre pesquisadores nesta área é procurar isolar fatores independentes (dimensões) que caracterizem os estilos de aprendizagem.

\footnotetext{
1 Eficácia educacional será definida aqui como "a medida da proporção em que os objetivos são alcançados" (Fundamentado na definição NBR 9241-11).
} 
Pask (1976) sugere que a combinação entre o estilo de aprendizagem e o método instrucional é condição necessária para o sucesso. Um estudo realizado por Allinson e Hayes (1996) analisando vinte trabalhos, sugere que a ponderação do estilo dos estudantes no projeto de experiências de aprendizagem poderá ser uma forma eficaz de otimizar a aprendizagem. Felder e Silverman (1988) afirmam que o uso dos modos verbal e não verbal simultaneamente reforça o aprendizado de todos os estudantes.

Assim, sob a óptica dos estilos de aprendizagem, a multimodalidade é uma forma de ganhar eficácia atendendo simultaneamente todos os perfis individuais dentro da dimensão verbal/não verbal.

\subsection{Teoria da Carga Cognitiva}

A Teoria da Carga Cognitiva (SWELLER, 1988) considera a capacidade de processamento necessária para a aquisição de esquemas ${ }^{2}$ e as questões relacionadas aos problemas decorrentes da sobrecarga cognitiva, que ocorre quando o sistema instrucional exige do estudante uma capacidade de processamento acima dos seus limites disponíveis.

Sweller (1988) e Paas et al (2003) apresentam princípios de projeto fundamentados na Teoria da Carga Cognitiva a partir da arquitetura cognitiva humana (Figura 01). São considerados três tipos de memória: memória sensorial, memória de trabalho e memória de longo prazo. Embora a memória de longo prazo possua capacidade virtualmente infinita, o ser humano somente pode acessar conscientemente a memória de trabalho, que é limitada quanto à sua capacidade e tempo de retenção. Tipicamente, o ser humano consegue lidar em sua memória de trabalho com cinco a nove elementos de informação simultaneamente (sete mais ou menos dois).

Elementos de informações novos (adquiridos através da memória sensorial) possuem ainda uma característica volátil, pois são perdidos cerca de 20 segundos após o último acesso.

Por outro lado, o número máximo de elementos de informação tratados simultaneamente se reduz para um valor entre dois e quatro quando há necessidade de algum tipo de processamento (organizar, comparar, ou qualquer outro tratamento) (Van Merriboer e

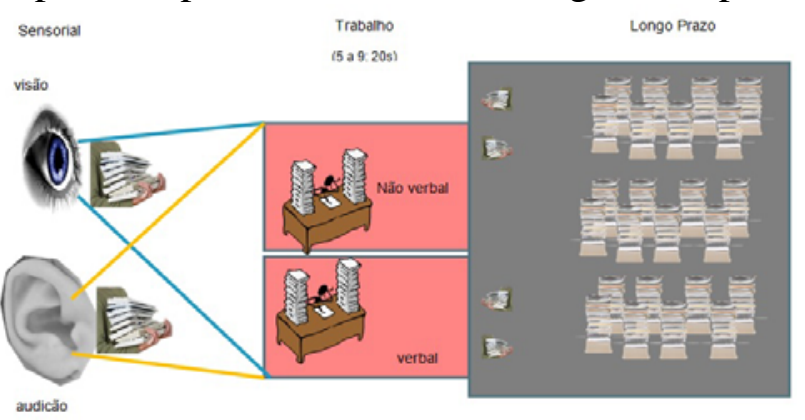

Figura 01. Arquitetura Cognitiva Humana (adaptado de Mayer et al, 2001) ${ }^{1}$ Sweller, 2010).

Baddeley (2010) destaca a existência de canais independentes de processamento de informações, associados aos modos sensoriais individuais: um canal para lidar com esquemas visuais ou espaciais e outro canal para lidar com as informações auditivas, especialmente a voz. O uso simultâneo destes dois canais possibilita o tratamento de um número maior de elementos de informação, o que está em consonância com as propostas de multimodalidade (Moreno e Mayer, 2007) e com o princípio de projeto multimídia

\footnotetext{
${ }^{2}$ Sweller (1988) define esquema como uma estrutura que permite identificar a situação problema como pertencente a uma determinada categoria de situações problema que normalmente necessita movimentos específicos.
} 
(Mayer, 2005). Assim, sob a óptica da teoria da carga cognitiva, a multimodalidade é uma forma de minimizar o risco de sobrecarga cognitiva.

\subsection{Multimodalidade, Interação e Aprendizagem}

A multimodalidade é um recurso com ganhos potenciais de eficácia, principalmente na aprendizagem receptiva, defendida por Ausubel (1978) como a forma mais simples e eficiente de aquisição de conhecimento. Ocorre que a aprendizagem receptiva requer um nível mais alto de maturidade cognitiva, no qual o estudante não se posiciona de forma passiva recebendo informação, mas de forma ativa.

No sistema de classificação de OAs multimodais, sugere-se também uma classificação quanto à interatividade, o que garantiria uma determinada proporção entre o aprendizado por recepção e o aprendizado por descoberta. Ainda neste contexto, podese compreender de forma clara o papel dos ambientes multimídias no processo de aprendizagem, por meio do seu conjunto de características. Ao se integrar diferentes recursos de multimídia em um único objeto, é necessário que alguns cuidados sejam tomados. Mayer (2001) descreve em sua pesquisa 3 aspectos que devem ser considerados: O pressuposto do canal duplo, que se caracteriza pelo fato do ser humano possuir dois canais de processamento de informação, o visual e verbal; A capacidade limitada, que descreve o limite de processamento das informações por cada canal, e; A aprendizagem ativa, que para ocorrer necessita a capacidade de processamento das informações em ambos os canais.

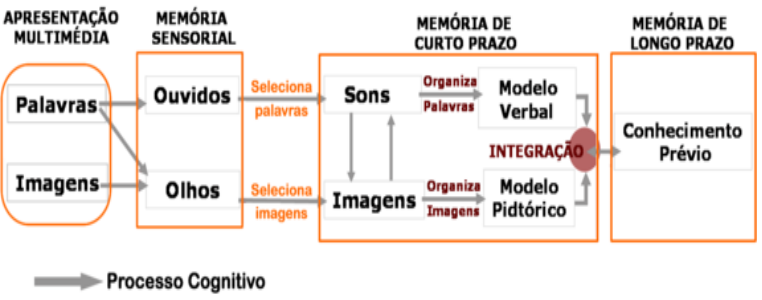

Figura 02. Processamento da Informação (Mayer, 2011)
Um dos resultados obtidos por Mayer em sua pesquisa apresenta sete princípios que devem ser respeitados no desenvolvimento de um material multimídia: Multimídia, Proximidade Espacial, Proximidade Temporal, Coerência, Modalidade, Redundância, Diferenças Individuais.

$\mathrm{O}$ reconhecimento dos sete princípios é requisito necessário ao se avaliar objetos de aprendizagem, vindo ao encontro desta proposta de pesquisa. Ao analisar a multimodalidade de um determinado software, pode-se reportar diretamente a elementos descritos em cada principio, a fim de vislumbrar seu efeito e, em algum momento, identificar prováveis classes em que estes poderão ser incluídos.

Porém, o que se pode observar é que os princípios de Mayer são de simples verificação, por parte do professor, quando pretende selecionar um Objeto de Aprendizagem adequado aos seus propósitos. Por outro lado, com relação à multimodalidade, esta análise torna-se um pouco mais complexa, o que reforça a necessidade do trabalho aqui relatado.

\section{Implementação}

Nas seções que seguem, será apresentado o método de classificação de objetos de aprendizagem propriamente dito, segundo a ótica de multimodalidade. 


\subsection{Análise dos elementos presentes em OAs}

A utilização de sistemas instrucionais multimídia que explorem o canal de áudio e a tela do computador para apresentar conceitos utilizando simultaneamente os modos verbal e não verbal (multimodalidade) traz consigo questionamentos relacionados à classe de multimodalidade utilizada.

Observa-se a existência de uma "zona cinzenta" (ver tabela 1) entre a informação verbal e a não verbal. Por exemplo, a exposição de um conteúdo narrado, utilizando o canal de áudio, é predominantemente verbal, mas não necessariamente apenas verbal. Isto porque uma narração pode ser monotônica ou carregada de recursos de oratória ou carga emocional (informações não verbais).

Da mesma forma, áudios não verbais podem ter algum significado individual ou cultural, trazendo consigo relações a conceitos, como se fossem palavras. Também as informações verbais apresentadas na tela (textos escritos) possuem uma carga de informação não

Tabela 1. Zona cinzenta verbal / não verbal

\begin{tabular}{|l|l|l|}
\hline & \multicolumn{1}{|c|}{ Áudio } & \multicolumn{1}{c|}{ Visual } \\
\hline Verbal & Palavras Faladas & Texto escrito \\
\hline \multirow{2}{*}{$\begin{array}{l}\text { Zona } \\
\text { Cinzenta }\end{array}$} & Entonação na voz & $\begin{array}{l}\text { Pontuação, grifos e sublinhados } \\
\text { Animações com palavras }\end{array}$ \\
\cline { 2 - 3 } & $\begin{array}{l}\text { Significados de áudios não } \\
\text { verbais }\end{array}$ & Ícones e ideogramas \\
\hline Não Verbal & Melodias, Efeitos Sonoros & Fotos, filmes \\
\hline
\end{tabular}
verbal (pontuação, fontes, grifados, sublinhados, etc.), e algumas informações não verbais se equiparam a palavras pelo fato de representarem conceitos (ideogramas, ícones, etc.).

$\mathrm{Na}$ prática, a eficácia da multimodalidade está intimamente relacionada ao significado dos áudios e imagens apresentados. Mayer e Sung (2012) efetuaram um estudo comparando o uso de sistemas sem imagem, com imagem instrutiva (imagens relacionadas ao conteúdo apresentado), com imagens sedutoras (muito interessantes, mas sem relacionamento com o conteúdo) e com imagens decorativas (neutras, mas não relacionadas ao conteúdo), e concluíram que os três tipos de imagem possuem efeitos motivacionais, mas apenas as imagens instrutivas melhoraram o aprendizado. Observaram também que imagens sedutoras possuem efeito negativo no aprendizado. Com base nisto, ressalta-se aqui que a aplicação do princípio da multimodalidade no projeto instrucional somente trará resultados positivos se também for aplicado o princípio da coerência.

\subsection{Proposta de um Método de Classificação}

A complexidade da mensuração da eficácia educacional leva à necessidade de que se desenvolvam alternativas mais simples, que possam ser utilizadas mesmo durante a fase de desenvolvimento do OA. A verificação de atendimento de um conjunto de princípios de projeto (MAYER 2001, 2005; MORENO \& MAYER, 2007; MAYER \& MORENO, 2003; GAGNÉ, 1987) pode ser uma alternativa prática capaz de estimar a eficácia de um determinado OA. Embora alguns princípios sejam de fácil verificação, como o princípio da continuidade espacial ou o princípio da continuidade temporal, observa-se que o princípio da multimodalidade possui nuances, devido a diversas possibilidades de multimodalidade. Por exemplo, a utilização de um áudio que possua conteúdo verbal e não verbal é um tipo de multimodalidade diferente de uma tela que apresente texto escrito acompanhado de imagens. Esta diversidade de situações levou à proposição de um sistema de classificação de OAs quanto ao tipo de multimodalidade, que parte do 
enquadramento do conteúdo audível e visual apresentado em um determinado trecho de aprendizagem receptiva segundo uma ou mais das seguintes categorias: Áudio Verbal: narrações em linguagem de domínio do público alvo; Áudio Não Verbal: qualquer informação sonora diferente de palavras faladas (efeitos sonoros, melodias, etc.); Visual Verbal: texto escrito apresentado na tela em linguagem de domínio do público alvo, e; Visual Não Verbal: informação visual apresentada na tela, diferente de texto escrito (imagens, animações, gráficos, etc.).

Foi considerada ainda a possibilidade (ou não) de interação do usuário com o objeto. Unindo todas estas possibilidades, percebe-se que qualquer OA multimodal encaixa-se necessariamente em alguma das linhas da tabela abaixo, na qual o " $\mathrm{X}$ " em um determinado item significa que o objeto atende àquela característica.

É importante observar que, já nesta primeira classificação, foram excluídas aquelas possibilidades em que o objeto não é multimodal, ou seja, não apresenta simultaneamente os modos verbal e não verbal nos estímulos visuais, auditivos, ou na combinação de ambos.

A partir da análise da Tabela 2, iniciou-se um processo de agrupamento de possibilidades que tivessem algumas características em comum, processo que resultou em sete classes, descritas na próxima seção.

\subsection{Redução e Normalização}

Com objetivo de tornar o método de classificação mais simples e prático de ser utilizado, após a criação da tabela 2 foram observadas as semelhanças e diferenças existentes entre cada uma das 18 possibilidades obtidas, chegando-se a uma divisão em sete classes, cada uma delas descrita a seguir:

- $\quad$ Classe 1: Multimodalidade

Dispersiva

Ocorre quando uma ou mais mídias

Tabela 2. Proposta inicial de classificação

\begin{tabular}{|c|c|c|c|c|c|}
\hline Linha & Áudio Verbal & Áudio Não Verbal & Visual Verbal & Visual Não Verbal & Interação \\
\hline 1 & & & X & X & \\
\hline 2 & & & X & X & X \\
\hline 3 & X & X & & & \\
\hline 4 & X & X & & & X \\
\hline 5 & & X & X & & \\
\hline 6 & & X & X & & X \\
\hline 7 & & X & X & X & \\
\hline 8 & & X & X & X & X \\
\hline 9 & X & & X & X & \\
\hline 10 & X & & X & X & X \\
\hline 11 & X & X & X & & \\
\hline 12 & X & X & X & & X \\
\hline 13 & X & X & X & X & \\
\hline 14 & X & X & X & X & X \\
\hline 15 & X & & & X & \\
\hline 16 & X & & & X & X \\
\hline 17 & X & X & & X & \\
\hline 18 & X & X & & X & X \\
\hline
\end{tabular}

apresentam conteúdo que não contribuam para o objetivo educacional proposto ou para a motivação do estudante, gerando carga cognitiva que não contribua para o aprendizado.

\section{- $\quad$ Classe 2 (linhas 1 a 4): Não Multimídia}

Esta segunda classe refere-se àqueles objetos que apresentam apenas áudio ou apenas imagem, porém em ambos os modos, para que possa ser considerado multimodal. Deuse a ela o nome de Não Multimídia justamente por tratar de situações em que se encontra apenas um estímulo sensorial, visual (uso da tela com textos escritos e imagens ou outro tipo de informação não verbal) ou auditivo (uso de canal de áudio com narração associada a algum tipo de informação não verbal audível, tais como efeitos sonoros ou melodias).

- $\quad$ Classe 3 (linhas 5 e 6): Texto Sonorizado. Como o próprio nome já diz, a classe 3 compreende os OAs que trazem apenas textos escritos acompanhados de um áudio não verbal. 
- $\quad$ Classe 4 (linhas 7 e 8): Texto Sonorizado Ilustrado. A classe 4, assim como a anterior, corresponde aos objetos que contêm textos escritos acompanhados de áudio, porém a diferença é que esta classe apresenta, ainda, estímulos visuais não verbais, ou seja, o texto sonorizado é acompanhado de uma figura, por exemplo.

- Classe 5 (linhas 9 a 14): Redundância Verbal. A classe "Redundância Verbal" foi assim denominada por conter informação verbal tanto no canal de áudio como na forma visual (texto narrado e escrito). Trata-se de uma alternativa menos eficaz do que a utilização de um único canal (ou narrado ou escrito - (MAYER et al, 2001)).

- $\quad$ Classe 6 (linhas 15 e 16): Multimídia Monotônico. Esta classe compreende basicamente aqueles objetos de aprendizagem que trazem a informação em forma de áudio verbal e visual não verbal, o que costuma ser bastante satisfatório no cumprimento de seus objetivos educacionais. Utilizou-se a expressão Monotônico para caracterizar o áudio verbal como uma narração pobre em recursos de oratória, ou imagens pouco elaboradas.

- $\quad$ Classe 7 (linhas 17 e 18): Multimídia Efetivo. A última classe, considerada a mais adequada segundo a classificação proposta, difere da classe anterior pela preocupação em acrescentar elementos afetivos coerentes com os objetivos educacionais e capazes de motivar o estudante para o aprendizado. São exemplos destes elementos afetivos o uso de efeitos sonoros, trilha sonora, design gráfico, e outros elementos que possam contribuir com a motivação sem dispersar a atenção do estudante.

Ressalta-se aqui que, em todas as classes, existem pelo menos duas linhas da Tabela 2, e que elas aparecem sempre em número par. Isto acontece porque, nas classes propostas, sempre existem duas possibilidades: interativo ou não interativo. Como o foco da proposta apresentada neste artigo é a multimodalidade, optou-se por realizar o agrupamento acima atentando mais para os aspectos áudio/visual/verbal/não verbal. Por outro lado, conforme já foi comentado, é sabido que a interação é uma característica bastante relevante no processo de aprendizagem através de OAs, motivo pelo qual ela não foi descartada.

\subsection{Aplicação e Validação do Método}

Como experimento prático, a fim de validar a proposta de classificação, utilizou-se um objeto de aprendizagem voltado para o ensino de bases numéricas ${ }^{3}$, aplicado a uma turma de graduação do curso de Engenharia de Computação, na disciplina de Introdução a Arquitetura de Computadores.

O objeto em questão dispõe de recursos visuais e áudios coerentes com os objetivos educacionais propostos. Os conteúdos teóricos são apresentados de forma segmentada; cada segmento inicia com uma narração acompanhada de animação; ao final do segmento o conteúdo narrado é apresentado na forma escrita. Este objeto foi inicialmente enquadrado como Multimídia Efetivo Interativo (classe 7). Observou-se, no entanto, que a proposição das questões apresentava simultaneamente a narração e textos escritos, caracterizando estes trechos como Redundância Verbal Interativo (classe 5).

O universo de indivíduos participantes do experimento consistiu em 20 alunos de terceiro semestre, os quais tiveram contato com o objeto de aprendizagem descrito

\footnotetext{
3 Objeto construído por um dos autores e disponibilizado no formato SCORM em um ambiente MOODLE institucional. (pode ser acessado também em http://www.engenhariaeletrica.ufrgs.br/public/numbin.2/)
} 
acima. O foco da pesquisa pontuou-se na identificação de aspectos da multimodalidade inerentes ao OA e qual o nível de conforto cognitivo dos estudantes durante sua utilização.

Ao classificar o OA predominantemente como Classe 7, percebe-se uma provável combinação adequada de diferentes mídias como áudio, vídeo e imagens, contudo é necessário observar o entendimento e concepção dos alunos sobre tal combinação. Com base nesta necessidade, um breve questionário com perguntas objetivas e subjetivas foi elaborado e disponibilizado aos estudantes, para que apresentassem suas considerações sobre como as mídias contidas no OA influenciaram na sua capacidade de interação com software. A Figura 3 apresenta um quadro com as perguntas elaboradas e aplicadas.

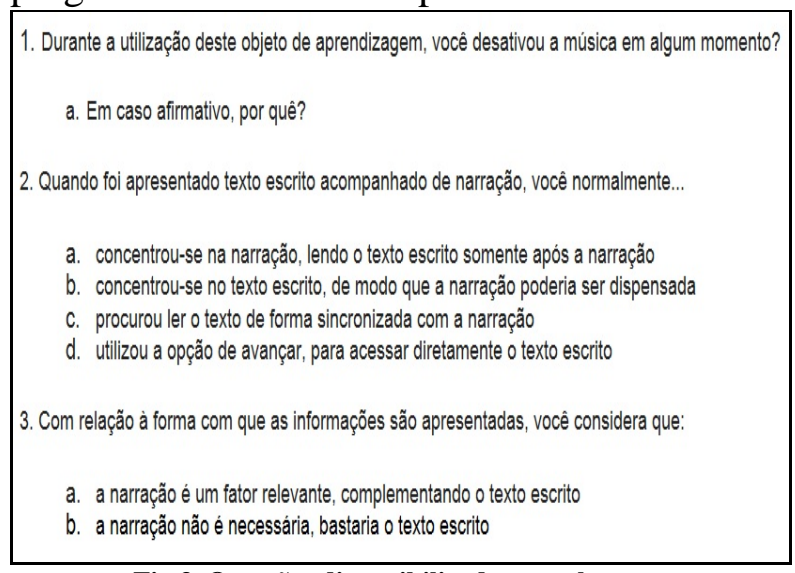

Fig 3. Questões disponibilizadas aos alunos
A tabulação dos resultados permitiu a observação de diferentes aspectos sobre multimodalidade. A maioria dos estudantes optou por desabilitar a música de fundo durante a execução do OA, conforme demonstrado no gráfico 01. Todos os alunos que desligaram a música durante a execução do objeto justificaram esta ação descrevendo a sobrecarga gerada, o que caracterizaria a música como um elemento de multimodalidade dispersiva. Percebe-se que, não obstante o OA oferecesse a opção de desabilitar a música, em vários

momentos o áudio sobrecarregou a capacidade de concentração dos alunos, conforme seus próprios depoimentos.
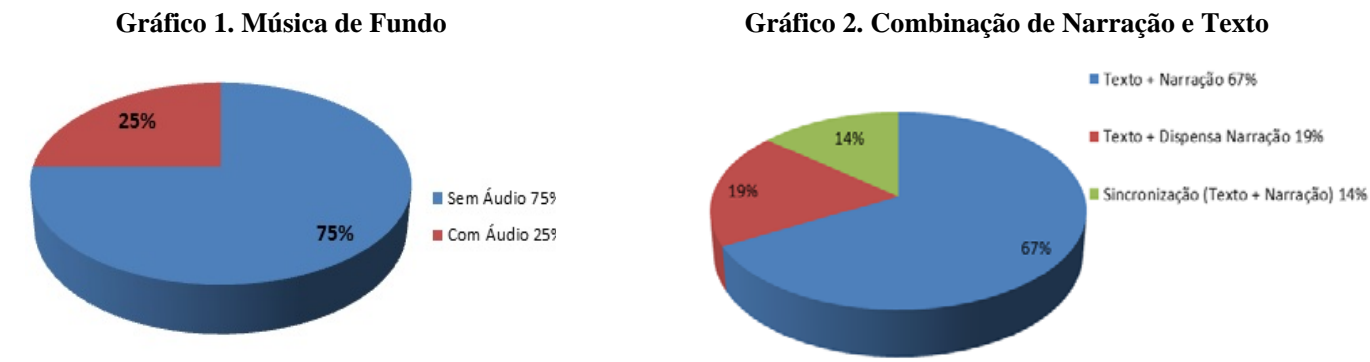

Em relação à apresentação do texto simultaneamente em dois formatos, áudio e escrito, disponíveis no OA, 67\% dos alunos descreveram que inicialmente se concentraram na narração e apenas depois leram os conteúdos escritos. O segundo grupo, formado por $19 \%$ dos participantes, afirmou que apenas se concentraram na leitura, dispensando a narração. Por fim, 14\% tentaram sincronizar suas leituras ao áudio do objeto. O gráfico 02 apresenta estes resultados.

A última questão visa observar qual a opinião dos estudantes quanto à apresentação simultânea de narração e texto escrito. Neste caso a maioria (81\%) descartou a narração, afirmando que apenas o texto é suficiente. O restante dos participantes (19\%) discorda, achando que a combinação de áudio e texto pode auxiliar na realização das tarefas, conforme apresentado no Gráfico 03. 
A interpretação dos resultados aponta para uma conclusão básica de que a narração integrada à apresentação de texto, em um mesmo momento, não é produtiva, fato que já havia sido apontado quando da descrição da classe 5 .

Esta hipótese foi comprovada,

Gráfico 3. Narração + Textos simultâneos

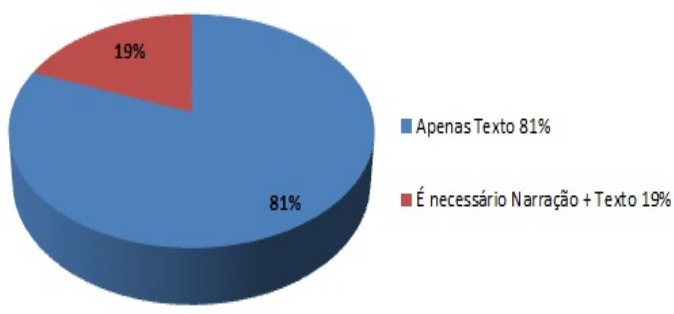
uma vez que a maior parte dos estudantes apontou para uma perda da concentração ao tentar sincronizar informações verbais vindas de ambas as mídias. Entende-se que o fato do $\mathrm{OA}$ adotado neste experimento utilizar diferentes mídias e por consequência estimular diferentes sentidos, traz uma condição que deve ser devidamente considerada no momento da utilização da classificação proposta, a fim de permitir uma correta pontuação dos Objetos de Aprendizagem avaliados, lembrando que o objetivo principal é permitir ao docente escolher de forma correta aplicações para serem utilizadas durante o processo de aprendizagem.

\section{Considerações Finais}

Este trabalho de pesquisa fundamentou-se basicamente no estudo sobre a multimodalidade, que pode estar presente em diferentes Objetos de Aprendizagem, e assumir um conjunto de diferentes combinações de mídias em uma mesma aplicação, como áudio de texto verbal, trilha sonora, recursos visuais, imagens, vídeos, entre outros. Este conjunto de funcionalidades acarreta na inserção de um alto grau de complexidade na identificação e seleção de um OA por parte do professor. Porém sabese que a escolha de um software de apoio à aprendizagem deve estar calcada em um conjunto de observações pertinentes, os quais balizem e garantam que o aluno consiga construir o conhecimento com a utilização de tais aplicações. Por este motivo, é necessário apoiar o professor nesta escolha e, neste sentido, a presente pesquisa foi desenvolvida com objetivo de trazer um método de classificação de OAs que seja simples, de fácil utilização, e que traga o mínimo possível de divergências entre diferentes utilizadores. Acredita-se que, com isto, foi possível trazer uma contribuição para a avaliação da eficácia do objeto.

A proposta partiu de uma classificação inicial com 18 possibilidades de enquadramento de um determinado $\mathrm{OA}$, com base no conjunto de mídias que poderiam estar presentes. A partir de uma minuciosa observação, apoiada por um relevante referencial teórico, este conjunto de possibilidades foi reduzido para apenas sete classes de multimodalidade, as quais apresentam efetiva relevância na identificação da multimodalidade presente em uma aplicação de apoio ao ensino. Como fundamentação para este estudo, uma avaliação da concepção de alunos sobre um respectivo OA foi realizada. Os resultados obtidos justificaram os principais pontos levantados durante a pesquisa, principalmente relacionados à questão da carga cognitiva que os estudantes conseguem assimilar durante uma atividade de estudo e da necessária coerência entre as mídias utilizadas. Neste contexto, a classificação proposta por esta pesquisa ficou corretamente alinhada com a multimodalidade intrínseca ao OA adotado, o que não garante a eficiência do software, mas assim permite descrevê-lo de acordo com o conjunto de mídias que ele possui. 
Com esta primeira aplicação, foi possível verificar alguns aspectos pontuais, tais como a questão da redundância verbal sugerida na Classe 5. Além disso, percebeu-se que, salvo algumas exceções, não será possível classificar um Objeto de Aprendizagem em apenas uma das 7 classes propostas, uma vez que ele deverá possuir várias telas, ou seções, diferentes e cada uma delas pode apresentar características de uma determinada classe. Como trabalhos futuros, pretende-se aplicar a classificação a uma variedade maior de OAs e observar a relação entre os grupos de aplicações que serão formados.

\section{Referências}

ALLINSON, C.W.; HAYES, J. (1996). The Cognitive Style Index: A measure of intuition-analysis for organisational research. Journal of Management Studies 33(1): 119-135.

AUSUBEL, D. P.; NOVAK, J. D.; HANESIAN, H. Psicologia educacional. 2. ed. Rio de Janeiro : Interamericana, 1978.

BADDELEY, A D. Working memory. Current Biology, v.23 (4), p.136-140, 2010.

BLOOM, B.S. Taxonomy of educational objectives: the classification of educational goals: New York; Toronto: Longmans, Green. 1956.

FELDER, R. M.; BRENT, R. Understanding student differences. Journal of Engineering Education, [s.l.], n. 94, v. 1, p. 57-72, 2005.

FELDER, R.M.; SILVERMAN, L.K. Learning and teaching styles in engineering education, Engineering Education, [s.l.], v. 78, n. 7, 1988, p. 674-681.

GAGNÉ, R. Instructional technology foundations. Hillsdale, NJ: Lawrence Erlbaum Assoc, 1987.

GRIMLEY, M; RIDING R. Individual differences and web-based learning. In: MOURLAS, C. et al.(eds.) Cognitive and emotional processes in web-based education: integrating human factors and personalization, Hershey: IGI Global, 2009, p. 209-228.

MAYER, R. E; SUNG E. When graphics improve liking but not learning from online lessons. Computers in Human Behavior, v. 28 (5), p. 1618-1625. 2012

MAYER, R. E. et al. Cognitive Constraints on Multimedia Learning: When Presenting More Material Results in Less Understanding. Journal of Educational Psychology, v. 93 (1). P 187-198. 2001.

MAYER, R. E. Introduction to multimedia learning. In: MAYER, R. E. (Ed.). The Cambridge Handbook of Multimedia Learning. New York: Cambridge University Press, 2005.

MAYER R E; MORENO R. Nine Ways to Reduce Cognitive Load in Multimedia Learning. Educational Psychology. V. 38 (1), p. 43-52, 2003.

MORENO, R.; MAYER R. Interactive multimodal learning environments. Educational Psychology Review,[n.l.], v. 19, p. 309-326, 2007.

Mussoi, E. M.; Flores, M. L. P.; Behar, P. A. (2010) Avaliação de Objetos de Aprendizagem. In: Congresso Iberoamericano de Informática Educativa, Santiago, Chile. Anais. Congresso Iberoamericano de Informática Educativa. Santiago: Jaime Sánchez, 2010.

NBR 9241-11. Requisitos Ergonômicos para Trabalho de Escritórios com Computadores. Rio de Janeiro, ABNT, Ago/2002.

PAAS F, RENKL, A, SWELLER, J. Cognitive Load Theory and Instructional Design: Recent Developments. Educational Psychologist. v 38, n l, 2003.

Pask. G. (1976). Styles and strategies of learning. British Journal of Educational Psychology 46: 128148.

Paivio, A. (1986). Mental representations: A dual coding approach. Oxford, England: Oxford University Press.

SWELLER, J. Cognitive load during problem solving: Effects on learning. Cognitive Science. 12, 257285, 1988

SWELLER, J. et al. Cognitive architecture and instructional design. Educational Psychology Review, 10, 251-296. 1998.

VAN MERRIENBOER, JJG; SWELLER, J: Cognitive load theory in health professional education: design principles and strategies. Medical Education, v. 44 p. 85-93, 2010.

WILEY, D. A. (2000) Connecting learning objects to instructional design theory: A definition, a metaphor, and a taxonomy. In: The Instructional Use of Learning Objects. Disponível em: $<$ http://reusability.org/read/chapters/wiley.doc>. Acesso em: 12 fev. 2013. 